\title{
Artifact Simulating Ventricular and Atrial Arrhythmia
}

\author{
Shoa-Lin Lin, M.D., Shih-Pu WANG, M.D., \\ Ghi-Woon Kong, M.D., and Mau-Song Chang, M.D.
}

SUMMARY

We describe a patient whose electrocardiograms showed ventricular tachycardia and atrial flutter which could be reproduced by arm movements. Careful review of the initiation and termination of the arrhythmia, the relation of the arrhythmia to body motion, and the associated symptoms and signs may be helpful to differentiate artifact from true arrhythmia.

\section{Key Words :}

Ventricular tachycardia Atrial flutter Arrhythmia

QUDDENLY developed ventricular tachycardia may be a poor prognostic $D$ sign. It may occasionally be produced by motion artifact. We present a case whose electrocardiogram showed an atypical ventricular tachycardia and atrial flutter over the electrocardiographic monitor in the coronary care unit. He did not have symptoms or signs of hemodynamic deterioration. These arrhythmias were eventually found to be induced by arm movements.

\section{Case Report}

The patient was a 63-year-old man who had history of old inferior and anterior myocardial infarction in March, 1989. Previous coronary angiography showed evidence of double vessel disease. Since he was initially reluctant to submit to percutaneous transluminal coronary angioplasty (PTCA), he had only supportive treatment at the outpatient clinic. Recently, because

From the Division of Cardiology, Department of Internal Medicine, Veterans General HospitalKaohsiung and Taipei, and the National Yang-Ming Medical College, Taipei, Taiwan, Republic of China.

Supported in part by the National Science Council, Taipei, Taiwan, Republic of China, Grant No. NSC 79-0412-B075-74.

Address for correspondence: Shoa-Lin Lin, M.D., Division of Gardiology, Veterans General Hospital-Kaohsiung, No. 386 Dar-Chung 1st Road, Kaohsiung City, Taiwan 80780, Republic of China.

Received for publication February 8, 1991.

Accepted June 20, 1991. 


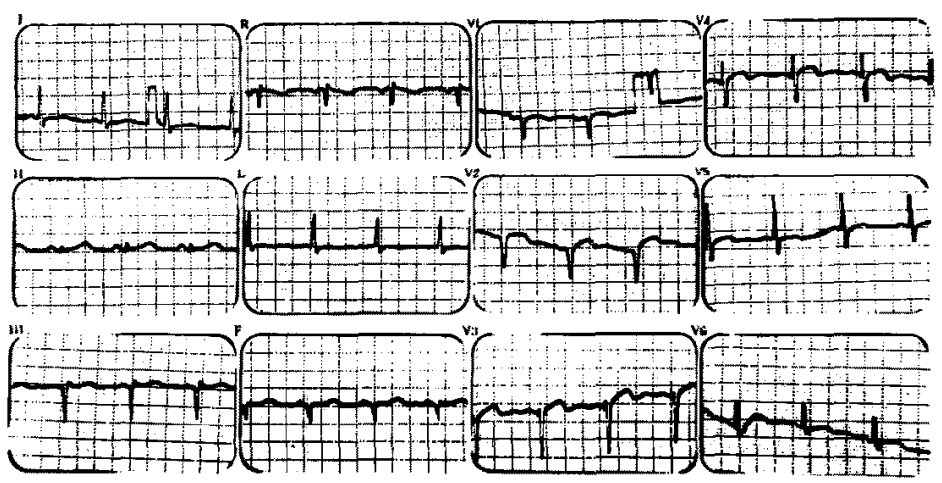

Fig. 1. Baseline electrocardiogram at admission. Old inferior and anteroseptal myocardial infarction were noted.

of increasing discomfort due to intermittent chest pain, he was admitted for coronary angioplasty. The coronary angiogram performed at admission disclosed a $90 \%$ stenosis of the mid-left anterior descending, a $50 \%$ stenosis of the first diagonal branch and an $80 \%$ stenosis of the distal circumflex coronary artery. The electrocardiogram showed normal sinus rhythm with old inferior and anteroseptal myocardial infarction (Fig. 1). Echocardiogram showed hypokinesis of the left ventricular anteroseptal and inferior walls associated with a small apical aneurysm. He received PTGA on November 24, 1990 ; the procedure was performed without difficulty with successful dilatation of the left anterior descending and left circumflex coronary arteries. After the procedure, he was observed in our coronary care unit beginning at about 11:00 a.m. His electrocardiogram (monitor lead II) was uneventful during the entire afternoon. The creatine phosphokinase was $73 \mathrm{U} / \mathrm{L}$ after PTCA. At 9:00 p.m., he was found to have an episode of ventricular tachycardia during tooth-brushing. Intermittent atrial flutter was also noted. There was no discomfort throughout the course and these arrhythmias stopped spontaneously after he finished brushing his teeth. The next morning, the same episodes of ventricular and atrial arrhythmia developed again while he was brushing his teeth. He was still free of any discomfort, and there was no blood pressure change. Serum creatine phosphokinase was $114 \mathrm{U} / \mathrm{L}$ in the morning. Initially, we thought that the repeated episodes of ventricular and atrial arrhythmias were probably due to his arm movements causing electrical artifacts in the electrocardiograms. To confirm this we suggested that he move his right arm. When the range of movement was greater, a similar electrocardiogram with atypical ventricular tachycardia recurred accordingly, and the atrial flutter developed again whenever arm movement was of a 

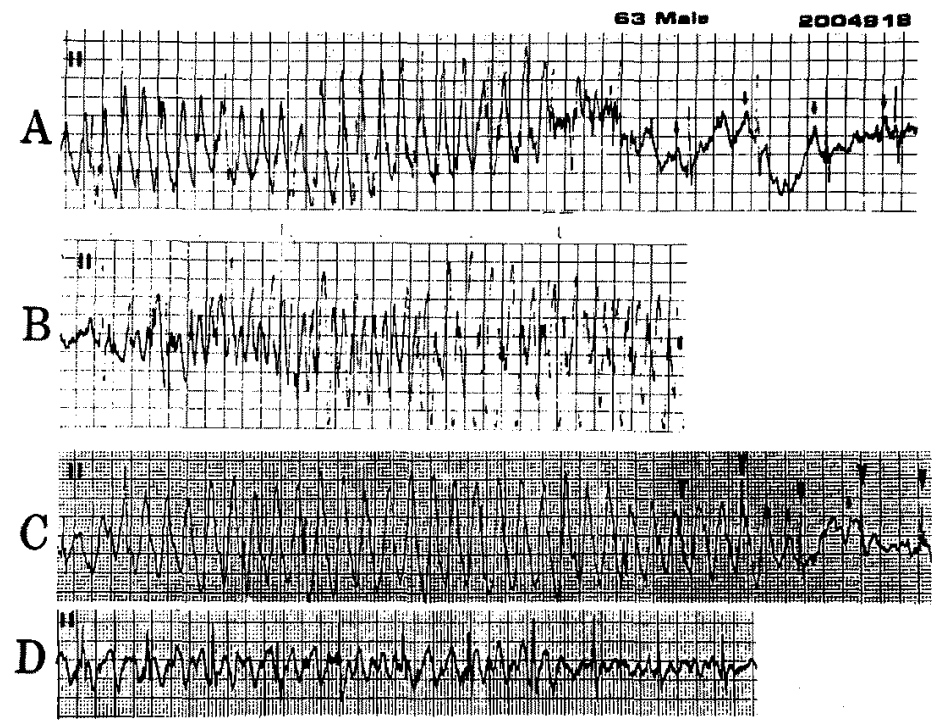

Fig. 2. Demonstrating the atypical ventricular tachycardia-like electrocardiograms terminated with sinus rhythm; the unstable baselines were found in the first several beats after the patient stopped arm movement (tracings $A$ and $C$ ). Small arrow indicates the $P$ wave and large arrow-head points to the QRS complex. The QRS complex could be found intermittently during and after the ventricular arrhythmia. Repeated episodes of ventricular arrhythmias were noted again when he began to wave his arm (tracing B). The atrial flutter-like electrocardiogram could be reproduced whenever arm movement was of a lesser degree (tracing D).

lesser extent (Fig. 2). The arrhythmia ceased immediately after he stopped waving his arm, only to be reproduced when he moved his arm again. Eventually, the ventricular and atrial arrhythmias were proved to be induced merely by his arm movements.

\section{DisaUssion}

Ventricular tachycardia is a life-threatening arrhythmia. It commonly occurs in a patient with organic heart disease, especially coronary artery disease. Hemodynamic deterioration may ensue if not treated promptly. It has been reported that a past history of myocardial infarction, congestive heart failure, or recent angina pectoris suggests that the wide-QRS tachycardia has a high likelihood of being ventricular in origin." ventricular tachycardias presenting as polymorphic wide-QRS tachycardia bring further difficulties in their interpretation and treatment. These include torsades de points, chaotic ventricular rhythm in a dying heart ${ }^{2)}$ and other 
ventricular tachyarrhythmias such as the wide QRS complex tachycardia in patients with global left ventricular ischemia in acute myocardial infarction ${ }^{3}$ and motion artifact. ${ }^{4}$

This case presented with episodes of atypical ventricular tachycardias induced by arm movements. A similar finding was also reported by Falk and his coworkers, ${ }^{4}$ ) who produced an atypical ventricular tachycardia by moving the electrocardiographic leads. They suggested that careful observation of the onset and termination may be helpful in distinguishing atypical ventricular tachycardia from artifact. This suggestion was also useful in discriminating between ventricular tachycardia and artifact in this patient. Additionally, we found that a narrow QRS complex could be observed at the end of the arrhythmia or appeared intermittently even during ventricular tachycardia-like electrocardiograms (Fig. 2, B and C). Observing the termination of ventricular tachycardia might demonstrate that the first several beats of sinus rhythm usually had an unstable baseline (Fig. 2, A). Narrow QRS complexes due to capture beats are occasionally observed during ventricular tachycardia. These can be easily differentiated from artifacts and should also be considered in the interpretation. Furthermore, the extent of arm movement with resulting electrical instability might cause variable electrocardiographic changes that range from atrial flutter-fibrillation to ventricular tachycardia-fibrillation. In this patient, the atrial flutter-like electrocardiograms were also detected and reproduced whenever the degree of arm movement was of a lesser extent (Fig. 2, D). Therefore, close examination of the relation of arrhythmia to the patient's motion could be helpful in distinguishing a real arrhythmia from an artifact.

The ventricular and atrial arrhythmias observed in this patient were proven to be induced by arm movements with lead jiggling. It may be possible that other movements such as combing hair, waving arms, moving the

Table 1. Factors Which May Be Helpful in Differentiating Artifact from Real Ventricular Arrhythmia

\begin{tabular}{|c|c|c|c|}
\hline & & Artifact & Arrhythmia \\
\hline I & Hemodynamic deterioration during arrhythmia & - & + \\
\hline 2 & Normal QRS complex mixed in arrhythmia & + &,+- \\
\hline 3 & Unstable baseline after termination of arrhythmia &,+- & - \\
\hline 4 & Association with body movement & + & - \\
\hline 5 & Reproducible with body motion & + & - \\
\hline 6 & $\begin{array}{l}\text { Excessive motion produces VT and lesser degree } \\
\text { of motion produces AF }\end{array}$ & + & - \\
\hline
\end{tabular}


body and general convulsions which move the electrocardiographic leads would produce similar arrhythmias as well.

In this patient, there were no symptoms or signs of hemodynamic change during the arrhythmia. Episodes of these ventricular and atrial arrhythmias related to arm movements could be found repeatedly. Thus, with any arrhythmia related to body motion but without symptoms or signs of hemodynamic change consideration should be given to the possibility of an artifact. In conclusion, we found that several factors may be useful to differentiate an artifact from a real arrhythmia (Table I). Careful review of these factors may avoid confusion in the electrocardiographic interpretation.

\section{References}

1. Baeman JM, Morady F, Dicarlo CA Jr, Buitleir M: Differentiation of ventricular tachycardia from supraventricular tachycardia with aberration: Value of the clinical history. Annals Emergency Med 16: 40, 1987

2. Chung EK: Ventricular arrhythmias. in Electrocardiography: Practical Applications with Vectorial Principles, ed by Chung EK, 3rd Ed, Appleton-Century-Crofts, Norwalk, p 24267,1985

3. Quinn TJ, Ezri MD, Denes P: ECG changes on global left ventricular ischemia simulating wide complex tachycardia. Chest 86:781, 1984

4. Falk RH, Knowlton AA: Atypical ventricular tachycardia or motion artifact (letter). Am J Cardiol 59: 924,1987 